\title{
Sentence Ranking for Document Indexing
}

\author{
Saptaditya Maiti ${ }^{1}$, Deba P. Mandal ${ }^{1}$, and Pabitra Mitra ${ }^{2}$ \\ 1 Machine Intelligence Unit, Indian Statistical Institute, Kolkata, India \\ 2 Dept. of Computer Science and Engineering, Indian Institute of Technology, \\ Kharagpur, India \\ \{saptaditya, dpmandal\}@isical.ac.in, \\ pabitra@gmail.com
}

\begin{abstract}
This article discusses a new document indexing scheme for information retrieval. For a structured (e.g., scientific) document, Pasi et al. proposed varying weights to different sections according to their importance in the document. This concept is extended here to unstructured documents. Each sentence in a document is initially assigned weight (significance in the document) with the help of a summarization technique. Accordingly, the term frequency of a term is decided as the sum of weights of the sentences the term belongs. The method is verified on a real life dataset using leading existing information retrieval models, and its performance has been found to be superior to conventional indexing schemes.
\end{abstract}

Keywords: information retrieval, document indexing, sentence ranking, relative entropy.

\section{Introduction}

Information retrieval is finding content (usually documents) of an unstructured nature (usually test) that satisfies an information need from within large collection 11. Information retrieval System (IRS) is based on relevance of a document to a query. Several methods have been proposed over years. The use of a strong query and a good retrieval method do not ensure good performance. In fact, the retrieval effectiveness is heavily affected by the model adopted for representing documents, since the retrieval mechanism performs a comparison between the user query and the representation of documents [2] 3].

The conventional indexing schemes assume that the information is homogeneously distributed in documents. Pasi et al. 2] 3] claimed that this assumption does not hold for a structured (e.g., scientific) document. The information content of a document varies from one section to another and hence, the terms in the more important sections should contribute more in retrieval methods. We argue that this is also true for unstructured documents. Usually, the flow of information in a given document is not uniform, which means that some parts are more important (informative) than others 4. We know that summarization techniques distinguish the more informative parts (i.e., sentences) from the less ones. 
In the present investigation, the weight/importance of a sentence in a document is initially measured with the help of a summarization technique, which is taken here as the relative entropy based method [5]. All of the terms in a sentence are assumed to have the same weight which is equal to the weight of the sentence. To obtain the term frequency (overall significance) of a term in a document, the weights for each of the occurrences of the term are aggregated. The effectiveness of the proposed indexing scheme is verified for a few leading retrieval models on FIRE dataset [6] and the results are found to be quite encouraging.

The rest of the paper is organized as follows. In section 2 , we describe the proposed indexing scheme. The implementation and effectiveness of the scheme is demonstrated in section 3. Section 4 finds the conclusions.

\section{Proposed Indexing Scheme}

As mentioned earlier, the proposed document indexing scheme is an extension of the model proposed by Pasi et al. 2] 3]. They suggested to provide different importance to different sections for indexing structured (e.g., scientific) documents. For example, in a scientific document, the terms in the 'Title' carry more information than those in other sections (e.g., 'abstract', 'introduction', etc.). In [2], different functions for different sections were proposed for providing varying weights/importance to the terms based on their belongingness in the document.

For the sake of extending the above model, we intend to impose structure to an unstructured document and then to allow varying weights to the terms based on their positions in the document. We find that extraction based text summarization methods analyze the importance/informativeness of different sentences in a document for selecting a few sentences as the summary of the document. Therefore, summarization techniques may be useful in finding the importance of the sentences in a document.

In the current investigation, each sentence in an unstructured document is visualized as to behave like a section of a structured document. With the help of a summarization technique, the informativeness of a sentence in a document is estimated, i.e., a weight is obtained for the sentence signifying its importance in the document.

The proposed indexing scheme consists of two parts. In the first part, weights of the sentences in a document are obtained using a text summarization method. The second part is concerned with the calculation of the term frequency of each of the terms in the document.

A) Sentence ranking: A text summarization method is used for ranking the sentences of a document. In this report, we have considered the relative entropy based summarization technique proposed by Kumar et al. [5].

This technique initially estimates the probability of a word $w$ in a sentence $S$ as

$$
P(w \mid S)=\frac{t f(w, S)}{|S|}
$$

where $t f(w, S)$ is the frequency of $w$ in $S$ and $|S|$ is the number of words in $S$. 
Similarly, the probability of $w$ in a document $D$ is estimated as

$$
P(w \mid D)=\frac{t f(w, D)}{|D|}
$$

where $t f(w, D)$ is the frequency of $w$ in $D$ and $|D|$ is the number of words in $D$.

A sentence $S$ is provided a weight based on its comparison to the document D. Comparison is done with relative entropy, i.e., KL-Divergence of $S$ with $D$ as

$$
K L_{S}=\sum_{w} P(w \mid S) \log \frac{P(w \mid S)}{P(w \mid D)} .
$$

As explained in [5], the importance of a sentence, $I_{S}$, is inversely proportional to $K L_{S}$, i.e.,

$$
I_{S}=\frac{1}{K L_{S}}
$$

We take $I_{S}$ as the weights of the sentences.

B) Term frequency: The significance of a sentence in a document is obtained previously. We assume that the significance is same for all the terms in a sentence. Accordingly, we assign the sentence weight to all of its terms. It may be realized that a term belonging to two different sentences may not have the same information content and hence the term may have different scores. Aggregating the scores of a term for all of its occurrences in a document, the term frequency of a term $t$ in $\mathrm{D}$ is calculated as

$$
t f(t, D)=\sum_{S_{i}} I_{S_{i}}
$$

The term frequency $t f(t, D)$ as in eqn.(5) reflects the importance of the term in document $D$ based not only on the number of occurrences but also on the information content for each of its occurrences. For example, suppose a document has the same number of occurrences for two terms $t_{1}$ and $t_{2}$, but $t_{1}$ has appeared in more important sentences than $t_{2}$. It is easy to realize here that $t_{1}$ is more significant than $t_{2}$. In such a case, the proposed scheme will find $t f$ value for $t_{1}$ higher than that of $t_{2}$ while the conventional indexing schemes will find the same $t f$ value for both $t_{1}$ and $t_{2}$. Hence, the proposed scheme should be a better indexing scheme than the existing ones which are based on raw counts of terms.

\section{Implementation and Results}

A new indexing scheme is described in the previous section. The effectiveness of the scheme is verified on the FIRE dataset [6] using Terrier platform [7]. We have considered some of the leading retrieval models available in Terrier, namely, Okapi BM25 [8], DFRBM25, InexpB2, InexpC2, IFB2, InL2 and BB2 [9]. A brief 
description of these models is provided in Table 1 It is to be noted that all the models have some parameters which need to be tuned. The parameter values mentioned in Table 1 against each model are the ones which are set in the Terrier platform [7] as default and we have used those values in implementing the models. The results are evaluated here based on Mean Average Precision (MAP).

Table 1. Description of retrieval models considered

\begin{tabular}{|l|l|r|}
\hline Model & Description & $\begin{array}{l}\text { Parameter } \\
\text { Values } \\
\text { Considered }\end{array}$ \\
\hline Okapi BM25 & The original BM25 probabilistic model & $\begin{array}{l}\mathrm{k} 1=1.2, \mathrm{k} 2=0, \\
\mathrm{k} 3=8, \mathrm{~b}=0.75\end{array}$ \\
\hline DFR_BM25 & The DFR version of BM25 & $\begin{array}{r}\mathrm{k} 1=1.2, \mathrm{k} 2=0, \\
\mathrm{k} 3=1000, \mathrm{c}=1\end{array}$ \\
\hline In_expB2 & $\begin{array}{l}\text { Inverse expected document frequency model for ran- } \\
\text { domness, the ratio of two Bernoulli's processes for first } \\
\text { normalisation, and Normalisation 2 for term frequency } \\
\text { normalisation }\end{array}$ & $\mathrm{c}=1$ \\
\hline In_expC2 & $\begin{array}{l}\text { Inverse expected document frequency model for ran- } \\
\text { domness, the ratio of two Bernoulli's processes for first } \\
\text { normalisation, and Normalisation 2 for term frequency } \\
\text { normalisation with natural logarithm }\end{array}$ & $\mathrm{c}=1$ \\
\hline IFB2 & $\begin{array}{l}\text { Inverse Term Frequency model for randomness, the ra- } \\
\text { tio of two Bernoulli's processes for first normalisation, } \\
\text { and Normalisation 2 for term frequency normalisation }\end{array}$ & $\mathrm{c}=1$ \\
\hline InL2 & $\begin{array}{l}\text { Inverse document frequency model for randomness, } \\
\text { Laplace succession for first normalisation, and Nor- } \\
\text { malisation 2 for term frequency normalisation }\end{array}$ & $\mathrm{c}=1$ \\
\hline BB2 & $\begin{array}{l}\text { Bose-Einstein model for randomness, the ratio of two } \\
\text { Bernoulli's processes for first normalisation, and Nor- } \\
\text { malisation 2 for term frequency normalisation }\end{array}$ & $\mathrm{c}=1$ \\
\hline
\end{tabular}

For, each of the models, we have experimented with both the conventional (based on raw counts) as well as proposed indexing schemes. In all the cases, the models with the proposed indexing scheme are found to perform better than that with the conventional indexing schemes. The MAP values of the said retrieval models with the conventional and the proposed indexing schemes are shown in Table 2. For more clarity, these results are shown graphically in Figure 1. 
Table 2. MAP of different models with conventional and proposed indexing schemes on FIRE dataset

\begin{tabular}{|c|c|c|c|c|c|c|c|}
\hline Indexing Scheme & BM25 & DFRBM25 & InExpB & InExpC & IF B2 & InL2 & BB2 \\
\hline Conventional & 0.5298 & 0.5304 & 0.5347 & 0.5299 & 0.5339 & 0.5420 & 0.5280 \\
\hline Proposed & 0.5732 & 0.5734 & 0.5798 & 0.5744 & 0.5803 & 0.5781 & 0.5747 \\
\hline
\end{tabular}

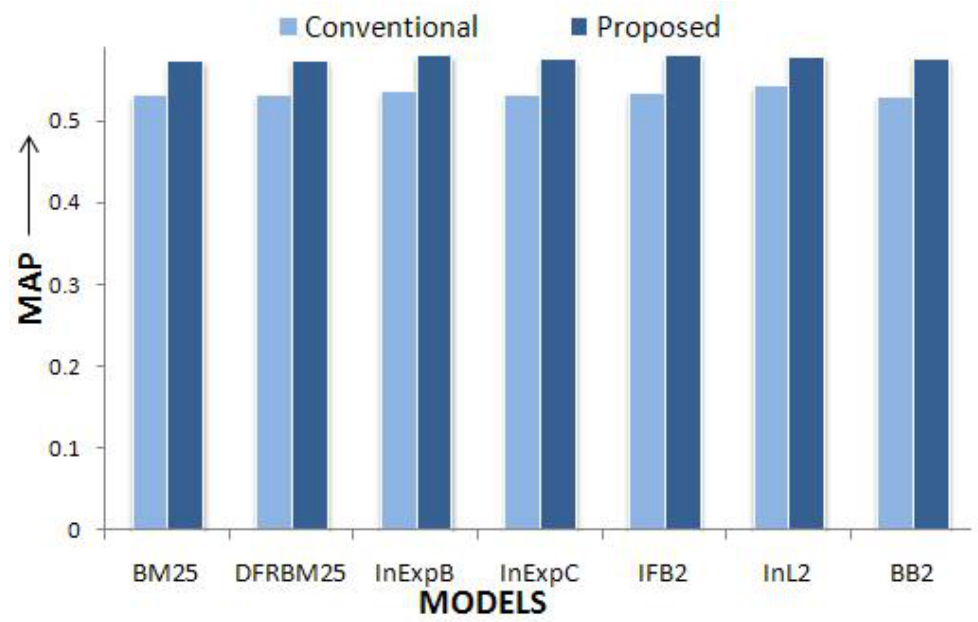

Fig. 1. MAP of different models with conventional and proposed indexing schemes

\section{Conclusions}

A new document indexing scheme is proposed in this article. Pasi et al. proposed different importance to terms in different sections of structured documents. We have extended this idea to unstructured documents. We assigned different scores to the sentences of a document using a summarization technique. It is to be mentioned here that we have taken relative entropy based summarization method for ranking the sentences, but one can take any other good summarization method. Instead of taking raw count for term frequency as in conventional indexing methods, we proposed the summation of scores of the sentences the term belongs to be the term frequency.

We have implemented this scheme in Terrier Platform and tested on FIRE dataset for a few leading information retrieval models. In all the cases considered, the performance of this scheme is found to be better than in the conventional term frequency. But, to make stronger claims the method is to be tested on more datasets. 


\section{References}

1. Manning, C.D., Raghavan, P., Schütze, H.: An Introduction to Information Retrieval. Cambridge University Press, New York (2008)

2. Bordogna, G., Pasi, G.: Controlling retrieval through a user-adaptive representation of documents. International Journal of Approximate Reasoning 12, 317-339 (1995)

3. Pasi, G.: Fuzzy Sets in Information Retrieval: State of the Art and Research Trends. In: Bustince, H., Herrera, F., Montero, J. (eds.) Fuzzy Sets and Their Extensions: Representation, Aggregation and Models, pp. 517-535. Springer, Heidelberg (2008)

4. Das, D., Martins, A.: A Survey on Automatic Text Summarization. Literature Survey for the Language and Statistics II Course at CMU (2007)

5. Kumar, C., Pingali, P., Varma, V.: A light-weight summarizer based on language model with relative entropy. In: Proc. of the ACM symposium on Applied Computing (SAC 2009). ACM, New York (2009)

6. Forum for Information Retrieval Evaluation (FIRE), http://www.isical.ac.in/ fire/

7. Ounis, I., Lioma, C., Macdonald, C., Plachouras, V.: Research Directions in Terrier: a Search Engine for Advanced Retrieval on the Web. Novatica/UPGRADE, Special Issue on Next Generation Web Search 8(1), 49-56 (2007)

8. Robertson, S.E., Walker, S.: Some simple approximations to the 2-poisson model for probabilistic weighted retrieval. In: Proc. of 17th Annual Int. Conf. on Research and Development in Information Retrieval, Dublin, pp. 232-241 (1994)

9. Amati, G.: Probability models for information retrieval based on divergence from randomness. Phd thesis, University of Glasgow (2003) 